\title{
Market Pricing for Matroid Rank Valuations
}

\author{
Kristóf Bérczi \\ MTA-ELTE Egerváry Research Group, Department of Operations Research, Eötvös Loránd \\ University, Budapest, Hungary \\ berkri@cs.elte.hu
}

\author{
Naonori Kakimura \\ kakimura@math.keio.ac.jp \\ Yusuke Kobayashi \\ Research Institute for Mathematical Sciences, Kyoto University, Japan \\ yusuke@kurims.kyoto.ac.jp
}

Department of Mathematics, Faculty of Science and Technology, Keio University, Yokohama, Japan

\begin{abstract}
In this paper, we study the problem of maximizing social welfare in combinatorial markets through pricing schemes. We consider the existence of prices that are capable to achieve optimal social welfare without a central tie-breaking coordinator. In the case of two buyers with matroid rank valuations, we give polynomial-time algorithms that always find such prices when one of the matroids is a simple partition matroid or both matroids are strongly base orderable. This result partially answers a question raised by Düetting and Végh in 2017. We further formalize a weighted variant of the conjecture of Düetting and Végh, and show that the weighted variant can be reduced to the unweighted one based on the weight-splitting theorem for weighted matroid intersection by Frank. We also show that a similar reduction technique works for $\mathrm{M}^{\natural}$-concave functions, or equivalently, for gross substitutes functions.
\end{abstract}

2012 ACM Subject Classification Theory of computation $\rightarrow$ Algorithmic mechanism design; Theory of computation $\rightarrow$ Computational pricing and auctions

Keywords and phrases Pricing schemes, Walrasian equilibrium, gross substitutes valuations, matroid rank functions

Digital Object Identifier 10.4230/LIPIcs.ISAAC.2020.39

Related Version A full version of the paper is available at [1] (https://arxiv.org/abs/2007.08759).

Funding This work was supported by the Research Institute for Mathematical Sciences, an International Joint Usage/Research Center located in Kyoto University.

Kristóf Bérczi: Supported by the János Bolyai Research Fellowship of the Hungarian Academy of Sciences, by the ÚNKP-19-4 New National Excellence Program of the Ministry for Innovation and Technology, and by projects no. NKFI-128673 and TKP2020-NKA-06 (National Challenges Subprogramme, "Application Domain Specific Highly Reliable IT Solutions") provided by the National Research, Development and Innovation Fund of Hungary.

Naonori Kakimura: Supported by JSPS KAKENHI grant numbers JP17K00028 and JP18H05291, Japan.

Yusuke Kobayashi: Supported by JSPS, KAKENHI grant numbers JP18H05291, JP19H05485, and JP20K11692, Japan.

\section{Introduction}

In this paper, we study the problem of maximizing social welfare in combinatorial markets through pricing schemes. Let us consider a combinatorial market consisting of indivisible goods and buyers, where each buyer has a valuation function that describes the buyer's preferences over the subsets of items. The goal is to allocate the items to buyers in such a way that the social welfare, that is, the total sum of the buyers' values, is maximized. Such an allocation can be found efficiently under reasonable assumptions on the valuations [27]. 
As an application of the Vickrey-Clarke-Groves (VCG) mechanism $[7,19,31]$ for welfare maximization, the VCG auction is another illustrious example. However, the problem becomes much more intricate if the optimal welfare ought to be achieved using simpler mechanisms employed in real world markets, such as pricing.

In a pricing scheme, the seller sets the item prices, and the utility of a buyer for a given bundle of items is defined as the value of the bundle with respect to the buyer's valuation, minus the total price of the items in the bundle. Ideally, the prices are set in such a way that there exists an allocation of the items to buyers in which the market clears and everyone receives a bundle that maximizes her utility. A pair of pricing and allocation possessing these properties is called a Walrasian equilibrium ${ }^{1}$, while we will refer to the price vector itself as Walrasian pricing. The fundamental notion of Walrasian equilibrium first appeared in [32], and the definition immediately implies that the allocation in a Walrasian equilibrium maximizes social welfare. Therefore, the problem might seem to be settled for markets that admit such an equilibrium.

Cohen-Addad et al. [8] observed that Walrasian prices alone are not sufficient to coordinate the market. The reason is that ties among different bundles have to be broken up carefully by a central coordinator, in a manner consistent with the corresponding optimal allocation. However, in real markets, buyers walk into the shop in an arbitrary sequential order and choose an arbitrary best bundle for themselves without caring about social optimum. In their paper, it is shown that the absence of a tie-braking rule may result in an arbitrarily bad allocation. In particular, no static prices can give more than $2 / 3$ of the social welfare when three buyers with unit-demand valuations arrive sequentially.

To overcome these difficulties, Cohen-Addad et al. [8] introduced the notion of dynamic pricing schemes. In this setting, the seller is allowed to dynamically update the prices between buyer arrivals. Achieving optimal social welfare based on dynamic pricing would be clearly possible if the order in which buyers arrive was known in advance. Nevertheless, determining an optimal dynamic pricing scheme is highly non-trivial when the prices need to be set before getting access to the preferences of the next buyer.

The main open problem in [8] asked whether any market with gross substitutes valuations has a dynamic pricing scheme that achieves optimal social welfare. A market with gross substitutes valuations is known to be an important class of markets having Walrasian prices [23]. It is worth noting that the existence of an optimal scheme reduces to the existence of an appropriate initial price vector; an optimal allocation then can be determined by induction. For a formal definition, we refer the reader to [2].

As a starting step towards understanding the general case, we consider the existence of a static pricing scheme for a two-buyer market with matroid rank valuations, because a matroid rank function is a fundamental example of gross substitutes valuations. Here, a matroid with a ground set $S$ and a base family $\mathcal{B}$ is denoted by $M=(S, \mathcal{B})$ and we denote $p(X):=\sum_{s \in X} p(s)$ for $p: S \rightarrow \mathbb{R}$ and $X \subseteq S$.

- Conjecture 1. Let $M_{1}=\left(S, \mathcal{B}_{1}\right)$ and $M_{2}=\left(S, \mathcal{B}_{2}\right)$ be matroids with rank functions $r_{1}$ and $r_{2}$, respectively. Then, there exists a function $p: S \rightarrow \mathbb{R}$ (called a price vector) satisfying the following conditions.

1. For any $B_{1} \in \arg \max _{X \subseteq S}\left(r_{1}(X)-p(X)\right)$ and for any $B_{2} \in \arg \max _{Y \subseteq S \backslash B_{1}}\left(r_{2}(Y)-p(Y)\right)$, $r_{1}\left(B_{1}\right)+r_{2}\left(B_{2}\right)=\max \left\{r_{1}(X)+r_{2}(Y) \mid X, Y \subseteq S, X \cap Y=\emptyset\right\}$.

2. For any $B_{2} \in \arg \max _{Y \subseteq S}\left(r_{2}(Y)-p(Y)\right)$ and for any $B_{1} \in \arg \max _{X \subseteq S \backslash B_{2}}\left(r_{1}(X)-p(X)\right)$, $r_{1}\left(B_{1}\right)+r_{2}\left(B_{2}\right)=\max \left\{r_{1}(X)+r_{2}(Y) \mid X, Y \subseteq S, X \cap Y=\emptyset\right\}$.

1 Walrasian equilibrium is often called competitive pricing, or market equilibrium in the literature. 
This conjecture can be interpreted as follows. There are two buyers and each buyer $i \in\{1,2\}$ has a valuation function $r_{i}$. If buyer $i$ comes to a shop first, then she chooses an arbitrary bundle $B_{i}$ that maximizes her utility $r_{i}-p$, and the second buyer chooses a best bundle in $S \backslash B_{i}$. The requirements mean that any choice of $B_{i}$ results in an allocation maximizing the social welfare. Thus, whoever comes first, we can achieve the optimal social welfare.

As we will see in Lemma 14, Conjecture 1 can be reduced to the following conjecture.

- Conjecture 2. Let $M_{1}=\left(S, \mathcal{B}_{1}\right)$ and $M_{2}=\left(S, \mathcal{B}_{2}\right)$ be matroids with a common ground set $S$ such that there exist disjoint bases $B_{1} \in \mathcal{B}_{1}$ and $B_{2} \in \mathcal{B}_{2}$ with $B_{1} \cup B_{2}=S$. Then, there exists a function $p: S \rightarrow \mathbb{R}$ (called a price vector) satisfying the following conditions.

1. For any $B_{1} \in \arg \min _{X \in \mathcal{B}_{1}} p(X)$, it holds that $S \backslash B_{1} \in \mathcal{B}_{2}$.

2. For any $B_{2} \in \arg \min _{X \in \mathcal{B}_{2}} p(X)$, it holds that $S \backslash B_{2} \in \mathcal{B}_{1}$.

In the conjecture, there are two buyers and each buyer $i \in\{1,2\}$ wants to buy a set of items that forms a basis in $\mathcal{B}_{i}$. If buyer $i$ comes to a shop first, then she chooses a cheapest set $B_{i}$ in $\mathcal{B}_{i}$ with an arbitrary tie-breaking rule. The requirements mean that, regardless of the choice of $B_{i}$, the remaining set $S \backslash B_{i}$ is a desired set for the other buyer.

Note that Conjecture 2 was first suggested by Dütting and Végh [12]. In their original conjecture, the price vector $p$ is chosen to have all different values, that is, $p\left(s_{1}\right) \neq p\left(s_{2}\right)$ for $s_{1} \neq s_{2}$, which implies that $B_{i} \in \arg \min _{X \in \mathcal{B}_{i}} p(X)$ is unique for $i=1,2$. However, this difference is not essential, because we can apply a perturbation to $p$ without affecting the requirements in Conjecture 2.

\section{Previous work}

The notion of Walrasian equilibrium dates back to 1874 [32], originally defined for divisible goods. In their analysis of the matching problem, Kelso and Crawford [23] introduced the so-called gross substitutes condition, and showed the existence of Walrasian prices for gross substitutes valuations. Gul and Stacchetti [20] later verified that, in a sense, this condition is necessary to ensure the existence of a Walrasian equilibrium. ${ }^{2}$

It was first observed by Cohen-Addad et al. [8] and Hsu et al. [21] that Walrasian prices are not sufficient to control the market, as ties must be broken in a coordinated fashion that is consistent with maximizing social welfare. A natural idea for resolving this issue would be trying to find Walrasian prices where ties do not occur. However, Hsu et al. showed that minimal Walrasian prices always induce ties. Even more, Cohen-Addad et al. proved that no static prices can give more than $2 / 3$ of the social welfare when three buyers with unit-demand valuations arrive sequentially. As a workaround, they proposed a dynamic pricing scheme for matching markets (i.e., unit-demand valuations), where the prices are updated between buyerarrivals based upon the current inventory without knowing the identity of the next buyer. On the negative side, they presented a market with coverage valuations where Walrasian prices do exist, but no dynamic pricing scheme can achieve the optimal social welfare. Meanwhile, $\mathrm{Hsu}$ et al. showed that, under certain conditions, minimal Walrasian equilibrium prices induce low over-demand and high welfare. Recently, Berger et al. [2] considered markets beyond unit-demand valuations, and gave a characterization of all optimal allocations in multi-demand markets. Based on this, they provided a polynomial-time algorithm for finding optimal dynamic prices up to three multi-demand buyers.

2 The simplest example of gross substitutes valuations are unit demand preferences, when each agent can enjoy at most one item. Gul and Stacchetti showed that gross substitutes preferences form the largest set containing unit demand preferences for which an existence theorem can be obtained. 
To overcome the limitations of Walrasian equilibrium, Feldman et al. [17] proposed a relaxation called combinatorial Walrasian equilibrium in which the seller can partition the items into indivisible bundles prior to sale, and they provided an algorithm that determines bundle prices obtaining at least half of the optimal social welfare.

Another line of research concentrated on posted-price mechanisms in online settings. As alternatives to optimal auctions, Blumrosen and Holenstein [3] studied posted-price mechanisms and dynamic auctions in Bayesian settings under the objective of maximizing revenue. They gave a characterization of the optimal revenue for general distributions, and provided algorithms that achieve the optimal solution. Chawla et al. $[4,5]$ developed a theory of sequential posted-price mechanisms, and provided constant-factor approximation algorithms for several multi-dimensional multi-unit auction problems and generalizations to matroid feasibility constraints. In [16], Feldman et el. verified the existence of prices that, in expectation, achieve at least half of the optimal social welfare for fractionally subadditive valuations, a class that includes all submodular functions. Dütting et al. $[10,11]$ provided a general framework for posted-price mechanisms in Bayesian settings. Chawla et al. [6] showed that static, anonymous bundle pricing mechanisms are useful when buyers' preferences have complementarities. Ezra et al. [15] provided upper and lower bounds on the largest fraction of the optimal social welfare that can be guaranteed with static prices for several classes of valuations, such as submodular, XOS, or subadditive. A setting related to online bipartite matching, called the Max-Min Greedy matching, was considered in [13].

\section{Our results}

In the present paper, we concentrate on combinatorial markets with two buyers having matroid rank valuations, where the matroid corresponding to buyer $i$ is denoted by $M_{i}=\left(S, \mathcal{B}_{i}\right)$ for $i=1,2$. Since this setting is reduced to Conjecture 2 , in which each buyer has to buy a set of items that forms a basis of a matroid, we focus on Conjecture 2 .

While Conjecture 2 remains open in general, we give polynomial-time ${ }^{3}$ algorithms for two important special cases. In the first one, one of the matroids is a partition matorid. Although partition matroids have relatively simple structure, finding the proper price vector $p$ is non-trivial even in this seemingly simple case.

- Theorem 3. Let $M_{1}$ be a partition matroid with partition classes of size at most 2 and with all-ones upper bound on the partition classes, and let $M_{2}$ be an arbitrary matroid. Then Conjectures 1 and 2 hold, and a price vector $p$ satisfying the conditions can be computed in polynomial time.

Next we consider strongly base orderable matroids, a class of matroids with distinctive structural properties. Roughly, in a strongly base orderable matroid, for any pair of bases, there exists a bijection between them satisfying a certain property (see Section 2 for the formal definition). Note that various matroids appearing in combinatorial and graph optimization problems belong to this class, such as partition, laminar, transversal matroids, or more generally, gammoids.

- Theorem 4. If both $M_{1}$ and $M_{2}$ are strongly base orderable, then Conjectures 1 and 2 hold. Furthermore, a price vector p satisfying the conditions can be computed in polynomial time if, for any pair of bases, the bijection between them can be computed in polynomial time.

3 In matroid algorithms, it is usually assumed that the matroids are accessed through independence oracles, and the complexity of an algorithm is measured by the number of oracle calls and other conventional elementary steps. 
Another contribution of this paper is to show the equivalence between Conjecture 2 and its weighted counterpart as below.

Conjecture 5. For $i \in\{1,2\}$, let $M_{i}=\left(S, \mathcal{B}_{i}\right)$ be a matroid and $w_{i}: S \rightarrow \mathbb{R}$ be a weight function. Assume that there exist disjoint bases $B_{1} \in \mathcal{B}_{1}$ and $B_{2} \in \mathcal{B}_{2}$ with $B_{1} \cup B_{2}=S$. Then, there exists a function $p: S \rightarrow \mathbb{R}$ satisfying the following conditions.

1. For any $B_{1} \in \arg \max _{X \in \mathcal{B}_{1}}\left(w_{1}(X)-p(X)\right)$, we have that $B_{1}$ is a maximizer of $w_{1}(X)+$ $w_{2}(S \backslash X)$ subject to $X \in \mathcal{B}_{1}$ and $S \backslash X \in \mathcal{B}_{2}$.

2. For any $B_{2} \in \arg \max _{X \in \mathcal{B}_{2}}\left(w_{2}(X)-p(X)\right)$, we have that $B_{2}$ is a maximizer of $w_{1}(S \backslash$ $X)+w_{2}(X)$ subject to $S \backslash X \in \mathcal{B}_{1}$ and $X \in \mathcal{B}_{2}$.

Clearly, Conjecture 2 is a special case of Conjecture 5; this follows easily by setting $w_{1} \equiv w_{2} \equiv 0$. Somewhat surprisingly, the reverse implication also holds for arbitrary matroids.

- Theorem 6. If Conjecture 2 is true, then Conjecture 5 is also true.

More generally, we prove that Theorem 6 can be generalized to the case with gross substitutes valuations, i.e., $\mathrm{M}^{\natural}$-concave functions. See the full version of this paper [1] for the details.

Based on Theorem 6 and the properties of partition and strongly base orderable matroids, we have the following corollaries.

- Corollary 7. Let $M_{1}$ be a partition matroid with partition classes of size at most 2 and with all-ones upper bound on the partition classes, and let $M_{2}$ be an arbitrary matroid. Then Conjecture 5 holds, and a price vector $p$ satisfying the conditions can be computed in polynomial time.

Corollary 8. If both $M_{1}$ and $M_{2}$ are strongly base orderable, then Conjecture 5 holds. Furthermore, a price vector $p$ satisfying the conditions can be computed in polynomial time if, for any pair of bases, the bijection between them can be computed in polynomial time.

\section{Paper organization}

The rest of the paper is organized as follows. Basic definitions and notation are given in Section 2. Theorems 3 and 4 are proved in Sections 3 and 4, respectively. The connection between unweighted and weighted variants of the problem is discussed in Section 5. We conclude the paper in Section 6.

\section{Preliminaries}

\section{Basic notation}

The sets of reals, non-negative reals, integers, and non-negative integers are denoted by $\mathbb{R}$, $\mathbb{R}_{+}, \mathbb{Z}$, and $\mathbb{Z}_{+}$, respectively. Let $S$ be a finite set. Given a subset $B \subseteq S$ and elements $x, y \in S$, we write $B-x+y$ for short to denote the set $(B \backslash\{x\}) \cup\{y\}$. The symmetric difference of two sets $X$ and $Y$ is $X \triangle Y:=(X \backslash Y) \cup(Y \backslash X)$. For a function $f: S \rightarrow \mathbb{R}$, we use $f(X):=\sum_{x \in X} f(x)$. For two vectors $x, y \in \mathbb{R}^{S}$, we denote $x \cdot y:=\sum_{s \in S} x(s) y(s)$. 


\section{Matroids and matroid intersection}

Matroids were introduced as an abstract generalization of linear independence in vector spaces $[28,33]$. A matroid $M$ is a pair $(S, \mathcal{I})$ where $S$ is the ground set of the matroid and $\mathcal{I} \subseteq 2^{S}$ is the family of independent sets satisfying the independence axioms: (I1) $\emptyset \in \mathcal{I}$, (I2) $X \subseteq Y \in \mathcal{I} \Rightarrow X \in \mathcal{I}$, and (I3) $X, Y \in \mathcal{I},|X|<|Y| \Rightarrow \exists e \in Y \backslash X$ s.t. $X+e \in \mathcal{I}$. A loop is an element that is non-independent on its own. The rank of a set $X \subseteq S$ is the maximum size of an independent set contained in $X$, and is denoted by $r(X)$. Here $r$ is called the rank function of $M$. Maximal independent sets of $M$ are called bases and their set is denoted by $\mathcal{B}$. Alternatively, matroids can be defined through the basis axioms: (B1) $\mathcal{B} \neq \emptyset$, and (B2) $B_{1}, B_{2} \in \mathcal{B}, x \in B_{1} \backslash B_{2} \Rightarrow \exists y \in B_{2} \backslash B_{1}$ s.t. $B_{1}-x+y \in \mathcal{B}$. In this paper, a matroid is denoted by a pair $(S, \mathcal{B})$, where $S$ is a ground set and $\mathcal{B}$ is a family of bases.

For a matroid $M=(S, \mathcal{B})$ and for $T \subseteq S$, deleting $T$ gives a matroid $M^{\prime}$ on the ground set $S \backslash T$ such that a subset of $S \backslash T$ is independent in $M^{\prime}$ if and only if it is independent in $M$. For $T \subseteq S$, contracting $T$ gives a matroid $M^{\prime}$ on the ground set $S \backslash T$ whose rank function is $r^{\prime}(X)=r(X \cup T)-r(T)$, where $r$ is the rank function of $M$. Adding a parallel copy of an element $s \in S$ gives a new matroid $M^{\prime}=\left(S^{\prime}, \mathcal{B}^{\prime}\right)$ on ground set $S^{\prime}=S+s^{\prime}$ where $\mathcal{B}^{\prime}=\left\{X \subseteq S^{\prime}\right.$ : either $X \in \mathcal{B}$, or $s \notin X, s^{\prime} \in X$ and $\left.X-s^{\prime}+s \in \mathcal{B}\right\}$. The direct sum $M_{1} \oplus M_{2}$ of matroids $M_{1}=\left(S_{1}, \mathcal{B}_{1}\right)$ and $M_{2}=\left(S_{2}, \mathcal{B}_{2}\right)$ on disjoint ground sets is a matroid $M=\left(S_{1} \cup S_{2}, \mathcal{B}\right)$ whose bases are the disjoint unions of a basis $M_{1}$ and a basis of $M_{2}$. The sum or union $M_{1}+M_{2}$ of $M_{1}=\left(S, \mathcal{B}_{1}\right)$ and $M_{2}=\left(S, \mathcal{B}_{2}\right)$ on the same ground set is a matroid $M=(S, \mathcal{B})$ whose independent sets are the disjoint unions of an independent set of $M_{1}$ and an independent set of $M_{2}$.

For a basis $B \in \mathcal{B}$, let us consider the bipartite graph $G=(S, E[B])$ defined by $E[B]:=$ $\{(x, y) \mid x \in B, y \in S \backslash B, B-x+y \in \mathcal{B}\}$. Krogdahl [24,25,26] verified the following statement (see also [30, Theorem 39.13]).

- Theorem 9 (Krogdahl). Let $M=(S, \mathcal{B})$ be a matroid and let $B \in \mathcal{B}$. Let $B^{\prime} \subseteq S$ be such that $|B|=\left|B^{\prime}\right|$ and $E[B]$ contains a unique perfect matching on $B \triangle B^{\prime}$. Then $B^{\prime} \in \mathcal{B}$.

In the weighted matroid intersection problem, we are given two matroids $M_{1}=\left(S, \mathcal{B}_{1}\right)$ and $M_{2}=\left(S, \mathcal{B}_{2}\right)$ on the same ground set together with a weight function $w: S \rightarrow \mathbb{R}$, and the goal is to find a common basis maximizing $w(B)$, that is, $B \in \arg \max \left\{w(B) \mid B \in \mathcal{B}_{1} \cap \mathcal{B}_{2}\right\}$. The celebrated weight-splitting theorem of Frank [18] gives a min-max relation for the weighted matroid intersection.

- Theorem 10 (Frank). The maximum w-weight of a common basis of $M_{1}=\left(S, \mathcal{B}_{1}\right)$ and $M_{2}=\left(S, \mathcal{B}_{2}\right)$ is equal to the minimum of $\max \left\{w_{1}(B) \mid B \in \mathcal{B}_{1}\right\}+\max \left\{w_{2}(B) \mid B \in \mathcal{B}_{2}\right\}$ subject to $w=w_{1}+w_{2}$. In particular, for an optimal weight-splitting $w=w_{1}+w_{2}$, it holds that $\arg \max \left\{w(B) \mid B \in \mathcal{B}_{1} \cap \mathcal{B}_{2}\right\}=\arg \max \left\{w_{1}(B) \mid B \in \mathcal{B}_{1}\right\} \cap \arg \max \left\{w_{2}(B) \mid B \in \mathcal{B}_{2}\right\}$.

A $k$-uniform matroid is a matroid $M=(S, \mathcal{B})$ where $\mathcal{B}=\{X \subseteq S|| X \mid=k\}$ for some $k \in \mathbb{Z}_{+}$. A partition matroid $M=(S, \mathcal{B})$ is the direct sum of uniform matroids, or in other words, $\mathcal{B}=\left\{X \subseteq S|| X \cap S_{i} \mid=k_{i}\right.$ for $\left.i=1, \ldots, q\right\}$ for some partition $S=S_{1} \cup \cdots \cup S_{q}$ of $S$ and $k_{i} \in \mathbb{Z}_{+}$for $i=1, \ldots, q$. Each $S_{i}$ is called a partition class. In the paper, we will work with partition matroids satisfying $\left|S_{i}\right| \leq 2$ and $k_{i}=1$ for $i=1, \ldots, q$.

For further details on matroids and the matroid intersection problem, we refer the reader to $[29,30]$. 


\section{Dual matroids}

The dual of a matroid $M=(S, \mathcal{B})$ is the matroid $M^{*}=\left(S, \mathcal{B}^{*}\right)$ where $\mathcal{B}^{*}=\left\{B^{*} \subseteq S \mid\right.$ $\left.S \backslash B^{*} \in \mathcal{B}\right\}$. Given one of the standard oracles for $M$, the same oracle for $M^{*}$ can be constructed as well.

We now rephrase Conjecture 2 by using dual matroids. Suppose that $M_{1}$ and $M_{2}$ are matroids as in Conjecture 2 and let $M_{2}^{*}=\left(S, \mathcal{B}_{2}^{*}\right)$ be the dual matroid of $M_{2}$. Then, we can see that $S \backslash B_{1} \in \mathcal{B}_{2}$ is equivalent to $B_{1} \in \mathcal{B}_{2}^{*}$, and $B_{2} \in \arg \min _{X \in \mathcal{B}_{2}} p(X)$ is equivalent to $S \backslash B_{2} \in \arg \max _{X \in \mathcal{B}_{2}^{*}} p(X)$. Therefore, by replacing $M_{2}$ and $S \backslash B_{2}$ with $M_{2}^{*}$ and $B_{2}$, respectively, Conjecture 2 is equivalent to the following conjecture.

- Conjecture 11. Let $M_{1}=\left(S, \mathcal{B}_{1}\right)$ and $M_{2}=\left(S, \mathcal{B}_{2}\right)$ be matroids with a common ground set $S$ such that there exists a common basis $B \in \mathcal{B}_{1} \cap \mathcal{B}_{2}$. Then, there exists a function $p: S \rightarrow \mathbb{R}$ satisfying the following conditions.

1. For any $B_{1} \in \arg \min _{X \in \mathcal{B}_{1}} p(X)$, it holds that $B_{1} \in \mathcal{B}_{2}$.

2. For any $B_{2} \in \arg \max _{X \in \mathcal{B}_{2}} p(X)$, it holds that $B_{2} \in \mathcal{B}_{1}$.

Conjecture 11 bears a lot of similarities with the problem of packing common bases in the intersection of two matroids. If $M_{1}$ and $M_{2}$ share two disjoint common bases, then setting the prices low on one of them and high on the other gives a desired $p$. If $S$ can be partitioned into two disjoint bases in both $M_{1}$ and $M_{2}$, then the statement may be reminiscent of Rota's famous conjecture concerning rearrangements of bases [22].

\section{Strongly base orderable matroids}

A matroid $M=(S, \mathcal{B})$ is strongly base orderable if for any two bases $B_{1}, B_{2} \in \mathcal{B}$, there exists a bijection $f: B_{1} \rightarrow B_{2}$ such that $\left(B_{1} \backslash X\right) \cup f(X) \in \mathcal{B}$ for any $X \subseteq B_{1}$, where we denote $f(X):=\{f(e) \mid e \in X\}$. Davies and McDiarmid [9] observed the following (see also [30, Theorem 42.13]).

- Theorem 12 (Davies and McDiarmid). Let $M_{1}=\left(S, \mathcal{B}_{1}\right)$ and $M_{2}=\left(S, \mathcal{B}_{2}\right)$ be strongly base orderable matroids. If $X \subseteq S$ can be partitioned into $k$ bases in both $M_{1}$ and $M_{2}$, then $X$ can be partitioned into $k$ common bases. Furthermore, such $k$ common bases can be computed in polynomial time if the bijection $f$ can be computed in polynomial time for any pair of bases.

The following technical lemma about strongly base orderable matroids will be used in the proof of Corollary 8 .

- Lemma 13. Let $M=(S, \mathcal{B})$ be a strongly base orderable matroid, $q: S \rightarrow \mathbb{R}$ be a function, and define a matroid $\hat{M}=(S, \hat{\mathcal{B}})$ by $\hat{\mathcal{B}}=\arg \max _{X \in \mathcal{B}} q(X)$. Then $\hat{M}$ is strongly base orderable.

Proof. Let $B_{1}, B_{2} \in \hat{\mathcal{B}}$. Since both $B_{1}$ and $B_{2}$ are bases of $M=(S, \mathcal{B})$, there exists a bijection $f: B_{1} \rightarrow B_{2}$ such that $\left(B_{1} \backslash X\right) \cup f(X) \in \mathcal{B}$ for any $X \subseteq B_{1}$. Since $q\left(B_{1}\right) \geq q\left(\left(B_{1} \backslash X\right) \cup f(X)\right)$ for any $X \subseteq B_{1}$ by $B_{1} \in \hat{\mathcal{B}}$, it holds that $q(X) \geq q(f(X))$. In particular, $q(x) \geq q(f(x))$ for any $x \in B_{1}$. Since $B_{2} \in \hat{\mathcal{B}}$, we obtain $q\left(B_{1}\right)=q\left(B_{2}\right)=q\left(f\left(B_{1}\right)\right)$, which shows that $q(x)=q(f(x))$ for any $x \in B_{1}$. Therefore, $q\left(B_{1}\right)=q\left(\left(B_{1} \backslash X\right) \cup f(X)\right)$ for any $X \subseteq B_{1}$, and hence $\left(B_{1} \backslash X\right) \cup f(X) \in \hat{\mathcal{B}}$. This shows that $\hat{M}$ is strongly base orderable.

\section{Market model}

In a combinatorial market, we are given a set $S$ of indivisible items and a set $J$ of buyers. Each buyer $i \in J$ has a valuation function $v_{i}: 2^{S} \rightarrow \mathbb{R}$ that describes the buyer's preferences over the subsets of items. Given prices $p: S \rightarrow \mathbb{R}$, the utility of buyer $i \in J$ for a subset 
$X \subseteq S$ is defined by $u_{i}(X)=v_{i}(X)-p(X)$. The buyers arrive in an undetermined order, and the next buyer always picks a subset of items that maximizes her utility. The goal is to set the prices in such a way that no matter which buyer arrives next, the final allocation of items maximizes the social welfare. In a dynamic pricing scheme, the prices can be updated between buyer arrivals based on the remaining sets of items and buyers.

We focus on the case of two buyers with matroid rank functions as valuations. Let $M_{1}=\left(S, \mathcal{B}_{1}\right)$ and $M_{2}=\left(S, \mathcal{B}_{2}\right)$ be matroids with rank functions $r_{1}$ and $r_{2}$, respectively. The valuation of agent $i$ is $r_{i}$ for $i=1,2$. The valuations are accessed through one of the standard matroid oracles (e.g. independence or rank oracle). As described in the introduction, this setting can be reduced to the case in which each buyer always chooses a basis that maximizes her utility, that is, Conjecture 1 can be reduced to Conjecture 2 .

- Lemma 14. If Conjecture 2 is true, then Conjecture 1 is also true.

Proof. Let $M_{1}=\left(S, \mathcal{B}_{1}\right)$ and $M_{2}=\left(S, \mathcal{B}_{2}\right)$ be matroids as in Conjecture 1 and let $\hat{B}_{1} \in \mathcal{B}_{1}$ and $\hat{B}_{2} \in \mathcal{B}_{2}$ be a pair of bases that maximizes $\left|\hat{B}_{1} \cup \hat{B}_{2}\right|$. For $i \in\{1,2\}$, let $M_{i}^{\prime}$ be the matroid obtained from $M_{i}$ by deleting $S \backslash\left(\hat{B}_{1} \cup \hat{B}_{2}\right)$ and contracting $\hat{B}_{1} \cap \hat{B}_{2}$. Then, $M_{1}^{\prime}=\left(S^{\prime}, \mathcal{B}_{1}^{\prime}\right)$ and $M_{2}^{\prime}=\left(S^{\prime}, \mathcal{B}_{2}^{\prime}\right)$ are matroids with a common ground set $S^{\prime}:=\left(\hat{B}_{1} \cup \hat{B}_{2}\right) \backslash\left(\hat{B}_{1} \cap \hat{B}_{2}\right)$ such that there exist disjoint bases $\hat{B}_{1} \backslash \hat{B}_{2} \in \mathcal{B}_{1}^{\prime}$ and $\hat{B}_{2} \backslash \hat{B}_{1} \in \mathcal{B}_{2}^{\prime}$ whose union is $S^{\prime}$. Hence, by assuming that Conjecture 2 is true, there exists a price vector $p^{\prime}: S^{\prime} \rightarrow \mathbb{R}$ with the following conditions.

1. For any $B_{1}^{\prime} \in \arg \min _{X \in \mathcal{B}_{1}^{\prime}} p^{\prime}(X)$, it holds that $S^{\prime} \backslash B_{1}^{\prime} \in \mathcal{B}_{2}^{\prime}$.

2. For any $B_{2}^{\prime} \in \arg \min _{X \in \mathcal{B}_{2}^{\prime}} p^{\prime}(X)$, it holds that $S^{\prime} \backslash B_{2}^{\prime} \in \mathcal{B}_{1}^{\prime}$.

We observe that we can modify the price vector $p^{\prime}$ so that $0<p^{\prime}(s)<1$ for every $s \in S^{\prime}$, by replacing $p^{\prime}(s)$ with $\alpha \cdot p^{\prime}(s)+\beta$ for some $\alpha>0$ and $\beta \in \mathbb{R}$. By using such a function $p^{\prime}$, define $p: S \rightarrow \mathbb{R}$ by

$$
p(s)= \begin{cases}p^{\prime}(s) & \text { if } s \in S^{\prime}, \\ 0 & \text { if } s \in \hat{B}_{1} \cap \hat{B}_{2}, \\ 1 & \text { if } s \in S \backslash\left(\hat{B}_{1} \cup \hat{B}_{2}\right) .\end{cases}
$$

For $B_{1} \in \arg \max _{X \subseteq S}\left(r_{1}(X)-p(X)\right)$, the definition of $p$ shows that $B_{1}=B_{1}^{\prime} \cup\left(\hat{B}_{1} \cap \hat{B}_{2}\right)$ for some $B_{1}^{\prime} \in \arg \min _{X \in \mathcal{B}_{1}^{\prime}} p^{\prime}(X)$. Since this implies $S^{\prime} \backslash B_{1}^{\prime} \in \mathcal{B}_{2}^{\prime}$, it holds that $S^{\prime} \backslash B_{1}^{\prime}$ is a maximal independent set of $M_{2}$ in $S \backslash B_{1}$ by the maximality of $\left|\hat{B}_{1} \cup \hat{B}_{2}\right|$. Therefore, if $B_{2} \in \arg \max _{Y \subseteq S \backslash B_{1}}\left(r_{2}(Y)-p(Y)\right)$, then $B_{2}=S^{\prime} \backslash B_{1}^{\prime}$ and hence

$$
\begin{aligned}
r_{1}\left(B_{1}\right)+r_{2}\left(B_{2}\right) & =\left|B_{1}^{\prime}\right|+\left|\hat{B}_{1} \cap \hat{B}_{2}\right|+\left|S^{\prime} \backslash B_{1}^{\prime}\right|=\left|\hat{B}_{1} \cup \hat{B}_{2}\right| \\
& =\max \left\{r_{1}(X)+r_{2}(Y) \mid X, Y \subseteq S, X \cap Y=\emptyset\right\},
\end{aligned}
$$

which shows the first requirement of Conjecture 1. The same argument works for $B_{2} \in$ $\arg \max _{X \subseteq S}\left(r_{2}(X)-p(X)\right)$. Therefore, $p$ satisfies the requirements in Conjecture 1 .

Note that a pair of bases $\hat{B}_{1} \in \mathcal{B}_{1}$ and $\hat{B}_{2} \in \mathcal{B}_{2}$ maximizing $\left|\hat{B}_{1} \cup \hat{B}_{2}\right|$ can be computed in polynomial time by applying a matroid intersection algorithm to $M_{1}$ and $M_{2}^{*}$. Note also that the price vector $p$ obtained in the above proof is not necessarily a Walrasian price.

We can consider a weighted variant of Conjecture 2 in which we are given weight functions $w_{1}: S \rightarrow \mathbb{R}$ and $w_{2}: S \rightarrow \mathbb{R}$. For a buyer $i \in\{1,2\}$ and for a basis $X \in \mathcal{B}_{i}$, the valuation $v_{i}(X)$ is defined as $w_{i}(X)$. Each buyer chooses a basis that maximizes her utility. Note that choosing a basis is a hard constraint, and hence we do not have to define $v_{i}(X)$ for $X \notin \mathcal{B}_{i}$. The goal is to find a price vector $p$ that achieves the optimal social welfare $\max \left\{w_{1}(X)+w_{2}(S \backslash X) \mid X \in \mathcal{B}_{1}, S \backslash X \in \mathcal{B}_{2}\right\}$. 
Recently, Berger et al. [2] investigated the existence of optimal dynamic pricing schemes for $k$-demand valuations. A valuation $v: 2^{S} \rightarrow \mathbb{R}_{+}$is $k$-demand if $v(X)=\max \left\{\sum_{s \in Z} v(s) \mid\right.$ $Z \subseteq X,|Z| \leq k\}$. Although this setting is similar to our weighted variant for $k$-uniform matroids, our results do not directly generalize their work because of our assumption on the buyers' choices.

\section{Partition matroids}

The aim of this section is to prove the existence of a required price vector $p$ for instances where $M_{1}$ is a partition matroid of special type.

- Theorem 3. Let $M_{1}$ be a partition matroid with partition classes of size at most 2 and with all-ones upper bound on the partition classes, and let $M_{2}$ be an arbitrary matroid. Then Conjectures 1 and 2 hold, and a price vector $p$ satisfying the conditions can be computed in polynomial time.

Proof. Since Conjectures 2 and 11 are equivalent by replacing $M_{2}$ with its dual $M_{2}^{*}$, we show Conjecture 11. Let $M_{1}=\left(S, \mathcal{B}_{1}\right)$ be a partition matroid defined by partition $S=S_{1} \cup \cdots \cup S_{q}$ where $\left|S_{i}\right| \leq 2$ for $i=1, \ldots, q$, that is, $\mathcal{B}_{1}=\left\{X \subseteq S|| X \cap S_{i} \mid=1\right.$ for $\left.i=1, \ldots, q\right\}$. Let $M_{2}=\left(S, \mathcal{B}_{2}\right)$ be an arbitrary matroid such that $M_{1}$ and $M_{2}$ have a common basis.

Let $B_{1} \in \mathcal{B}_{1} \cap \mathcal{B}_{2}$ be an arbitrary common basis. Take another common basis $B_{2} \in \mathcal{B}_{1} \cap \mathcal{B}_{2}$ (possibly $B_{2}=B_{1}$ ) such that $\left|B_{1} \cap B_{2}\right|$ is minimized. We consider a bipartite digraph $D=(V, E)$ defined by

$$
\begin{aligned}
V= & \left(B_{1} \cap B_{2}\right) \cup\left(S \backslash\left(B_{1} \cup B_{2}\right)\right), \\
E= & \left\{(x, y) \mid x \in B_{1} \cap B_{2}, y \in S \backslash\left(B_{1} \cup B_{2}\right), B_{1}-x+y \in \mathcal{B}_{1}\right\} \\
& \cup\left\{(y, x) \mid x \in B_{1} \cap B_{2}, y \in S \backslash\left(B_{1} \cup B_{2}\right), B_{2}-x+y \in \mathcal{B}_{2}\right\} .
\end{aligned}
$$

$\triangleright$ Claim 15. The digraph $D$ is acyclic.

Proof. Let $x \in B_{1} \cap B_{2}$ and $y \in S \backslash\left(B_{1} \cup B_{2}\right)$. As $M_{1}$ is defined on a partition consisting of classes of size at most $2, B-x+y \in \mathcal{B}_{1}$ implies that $\{x, y\}$ is one of the partition classes. This implies that $B_{1}-x+y \in \mathcal{B}_{1}$ if and only if $B_{2}+x-y \in \mathcal{B}_{1}$.

Now suppose to the contrary that $D$ contains a dicycle. Choose a dicycle $C$ with the smallest number of vertices, which implies that $C$ has no chord. Then, $B_{2}^{\prime}:=B_{2} \triangle V(C)$ is a common basis of $M_{1}$ and $M_{2}$ by the above observation and Theorem 9. Since $\left|B_{1} \cap B_{2}^{\prime}\right|<$ $\left|B_{1} \cap B_{2}\right|$, this contradicts that $\left|B_{1} \cap B_{2}\right|$ is minimized.

Let $n=|S|$. We now consider a function $p: S \rightarrow \mathbb{R}$ satisfying the following: $p(x):=0$ for $x \in B_{1} \backslash B_{2}, p(x):=n+1$ for $x \in B_{2} \backslash B_{1}, p(x)$ are distinct values in $\{1,2, \ldots, n\}$ for $x \in V$, and $p(x)<p(y)$ for $(x, y) \in E$. Note that such a function exists by Claim 15 , which can be found easily by the topological sorting. In what follows, we show that $p$ satisfies that $\arg \min _{X \in \mathcal{B}_{1}} p(X)=\left\{B_{1}\right\}$ and $\arg \max _{X \in \mathcal{B}_{2}} p(X)=\left\{B_{2}\right\}$

$\triangleright$ Claim 16. $\arg \min _{X \in \mathcal{B}_{1}} p(X)=\left\{B_{1}\right\}$ and $\arg \max _{X \in \mathcal{B}_{2}} p(X)=\left\{B_{2}\right\}$.

Proof. For a non-negative integer $k$, let $S_{k}:=\{x \in S \mid p(x) \leq k\}$ and let $I_{k}$ be a minimizer of $p(X)$ subject to $X$ being a maximal independent set of $M_{1}$ and $X \subseteq S_{k}$. Note that $I_{k}$ can be computed by a greedy algorithm. Since $S_{n}$ contains a basis $B_{1}$, the greedy algorithm chooses no element in $B_{2} \backslash B_{1}$, which means that $I_{k} \cap\left(B_{2} \backslash B_{1}\right)=\emptyset$ for every $k$. We also note that $I_{k}$ is uniquely determined for each $k$, since the $p(x)$ 's are distinct for $x \in V$. 
We show that $I_{k}=B_{1} \cap S_{k}$ for every $k$ by induction on $k$. Since $I_{0}=B_{1} \backslash B_{2}$, it is obvious that $I_{0}=B_{1} \cap S_{0}$. Fix $k \geq 1$ and assume that $I_{k-1}=B_{1} \cap S_{k-1}$. Then, we have the following.

- If there exists $x \in B_{1} \cap B_{2}$ with $p(x)=k$, then $I_{k}=I_{k-1}+x$, and hence $I_{k}=B_{1} \cap S_{k}$.

- Suppose that there exists $y \in S \backslash\left(B_{1} \cup B_{2}\right)$ with $p(y)=k$. We show that $I_{k-1}+y$ is not independent in $M_{1}$. Suppose to the contrary that $I_{k-1}+y$ is independent. Then, there exists $x \in B_{1} \backslash I_{k-1}$ such that $B_{1}-x+y \in \mathcal{B}_{1}$, and hence $(x, y) \in E$. By the choice of $p$, we obtain $p(x)<p(y)$, i.e., $x \in S_{k-1}$. This contradicts $x \in B_{1} \backslash I_{k-1}$, because $S_{k-1} \cap\left(B_{1} \backslash I_{k-1}\right)=\emptyset$ by the induction hypothesis. Therefore, $I_{k-1}+y$ is not independent in $M_{1}$, which shows that $I_{k}=I_{k-1}$ and $I_{k}=B_{1} \cap S_{k}$.

- If there exists no $x \in V$ with $p(x)=k$, then $I_{k}=I_{k-1}$, and hence $I_{k}=B_{1} \cap S_{k}$.

Therefore, $I_{k}=B_{1} \cap S_{k}$ holds for every $k$ by induction. This shows that $I_{n+1}=B_{1} \cap S_{n+1}=$ $B_{1}$, and hence $\arg \min _{X \in \mathcal{B}_{1}} p(X)=\left\{I_{n+1}\right\}=\left\{B_{1}\right\}$.

By a similar argument, we obtain $\arg \max _{X \in \mathcal{B}_{2}} p(X)=\left\{B_{2}\right\}$.

Since $B_{1}, B_{2} \in \mathcal{B}_{1} \cap \mathcal{B}_{2}$, this claim shows that $p$ satisfies the requirements in Conjecture 11 . Thus, Conjecture 11 holds, and hence Conjecture 2 also holds.

This together with Lemma 14 shows that Conjecture 1 also holds. Note that, in the proof of Lemma 14, we modify given matroids by deleting and contracting some elements, but this modification does not affect the assumption on $M_{1}$. That is, if $M_{1}$ is a partition matroid with partition classes of size at most 2 and with all-ones upper bound on the partition classes, then the obtained matroid $M_{1}^{\prime}$ is also a partition matroid of this type.

- Remark 17. Note that in the proof of Theorem 3, we fixed the basis $B_{1} \in \mathcal{B}_{1}$ arbitrarily. That is, for any $B_{1} \in \mathcal{B}_{1}$, the optimal price vector $p$ can be set in such a way that the maximum utility of the buyer corresponding to $M_{1}$ is attained on $B_{1}$. It is not difficult to see that the analogous statement holds for any basis $B_{2} \in \mathcal{B}_{2}$.

- Remark 18. Even when $\mathcal{B}_{1}$ is a base family of a partition matroid as in Theorem 3, if $\mathcal{B}_{2}$ is an arbitrary set family of $S$, then the requirements in Conjecture 2 do not necessarily hold. To see this, suppose that $S=\{1,2,3,4\}, \mathcal{B}_{1}=\{\{1,3\},\{1,4\},\{2,3\},\{2,4\}\}$, and $\mathcal{B}_{2}=\{\{2,4\},\{1,2\},\{3,4\}\}$. Then, $\left(B_{1}, B_{2}\right)=(\{1,3\},\{2,4\})$ is a unique pair of disjoint sets such that $B_{1} \in \mathcal{B}_{1}, B_{2} \in \mathcal{B}_{2}$, and $B_{1} \cup B_{2}=S$. If $p$ satisfies the requirements in Conjecture 2, then $p(1)<p(2)$ and $p(3)<p(4)$ hold by the first requirement and $p(4)<p(1)$ and $p(2)<p(3)$ hold by the second requirement. This shows that such $p$ does not exist.

\section{Strongly base orderable matroids}

In this section, we show that Conjectures 1 and 2 hold for strongly base orderable matroids. The proof is based on a similar approach to that of Theorem 3. Nevertheless, there are small but crucial differences.

- Theorem 4. If both $M_{1}$ and $M_{2}$ are strongly base orderable, then Conjectures 1 and 2 hold. Furthermore, a price vector $p$ satisfying the conditions can be computed in polynomial time if, for any pair of bases, the bijection between them can be computed in polynomial time.

Proof. In order to show Conjecture 2, we first show Conjecture 11 under the assumption that $M_{1}$ and $M_{2}$ are strongly base orderable. Let $M_{1}=\left(S, \mathcal{B}_{1}\right)$ and $M_{2}=\left(S, \mathcal{B}_{2}\right)$ be strongly base orderable matroids that have a common basis. We take two common bases $B_{1}, B_{2} \in \mathcal{B}_{1} \cap \mathcal{B}_{2}$ (possibly $B_{1}=B_{2}$ ) such that $\left|B_{1} \cap B_{2}\right|$ is minimized. For each element $x \in S$, we add a parallel copy $x^{\prime}$ of $x$ to the matroid $M_{i}$ and denote the matroid thus 
obtained by $M_{i}^{+}=\left(S \cup S^{\prime}, \mathcal{B}_{i}^{+}\right)$for $i \in\{1,2\}$. We denote $X^{\prime}:=\left\{x^{\prime} \mid x \in X\right\}$ for $X \subseteq S$. Let $2 M_{i}^{+}=\left(S \cup S^{\prime}, 2 \mathcal{B}_{i}^{+}\right)$be the sum of two copies of $M_{i}^{+}$. As $M_{i}^{+}$clearly has two disjoint bases, we have $2 \mathcal{B}_{i}^{+}:=\left\{Y_{1} \cup Y_{2} \mid Y_{1}\right.$ and $Y_{2}$ are disjoint bases of $\left.M_{i}^{+}\right\}$.

$\triangleright$ Claim 19. For $i \in\{1,2\}, 2 M_{i}^{+}$is a strongly base orderable matroid.

Proof. Fix $i \in\{1,2\}$. We can easily see that $M_{i}^{+}$is strongly base orderable. Suppose that we are given two bases $X_{1}, X_{2} \in 2 \mathcal{B}_{i}^{+}$, and suppose also that $X_{1}=Y_{1}^{1} \cup Y_{1}^{2}$ and $X_{2}=Y_{2}^{1} \cup Y_{2}^{2}$, where $Y_{1}^{1}, Y_{1}^{2}, Y_{2}^{1}, Y_{2}^{2} \in \mathcal{B}_{i}^{+}$. Since $M_{i}^{+}$is strongly base orderable, for $j \in\{1,2\}$, there exists a bijection $f_{j}: Y_{1}^{j} \rightarrow Y_{2}^{j}$ such that $\left(Y_{1}^{j} \backslash X\right) \cup f_{j}(X) \in \mathcal{B}_{i}^{+}$for any $X \subseteq Y_{1}^{j}$. Then, $f_{1}$ and $f_{2}$ naturally define a bijection $f: X_{1} \rightarrow X_{2}$ such that $\left(X_{1} \backslash X\right) \cup f(X) \in 2 \mathcal{B}_{i}^{+}$for any $X \subseteq X_{1}$. This shows that $2 M_{i}^{+}$is strongly base orderable.

Let $X_{0}:=\left(B_{1} \cup B_{2}\right) \cup\left(B_{1} \cap B_{2}\right)^{\prime}$. Then, $X_{0}$ is a common basis of $2 M_{1}^{+}$and $2 M_{2}^{+}$. We consider a bipartite digraph $D^{+}=\left(V, E^{+}\right)$defined by

$$
\begin{aligned}
V= & \left(B_{1} \cap B_{2}\right) \cup\left(S \backslash\left(B_{1} \cup B_{2}\right)\right), \\
E^{+}= & \left\{(x, y) \mid x \in B_{1} \cap B_{2}, y \in S \backslash\left(B_{1} \cup B_{2}\right), X_{0}-x+y \in 2 \mathcal{B}_{1}^{+}\right\} \\
& \cup\left\{(y, x) \mid x \in B_{1} \cap B_{2}, y \in S \backslash\left(B_{1} \cup B_{2}\right), X_{0}-x+y \in 2 \mathcal{B}_{2}^{+}\right\} .
\end{aligned}
$$

$\triangleright$ Claim 20. The digraph $D^{+}$is acyclic.

Proof. Suppose to the contrary that $D^{+}$contains a dicycle. Choose a dicycle $C$ with the smallest number of vertices, which implies that $C$ has no chord. Then, $X_{0} \triangle V(C)$ is a common basis of $2 M_{1}^{+}$and $2 M_{2}^{+}$by Theorem 9. By Theorem 12 and Claim 19, $X_{0} \triangle V(C)$ can be partitioned into two common bases of $M_{1}^{+}$and $M_{2}^{+}$. Let $\tilde{B}_{1}$ and $\tilde{B}_{2}$ be the sets in $S$ corresponding to these common bases. Then, $\tilde{B}_{1}, \tilde{B}_{2} \in \mathcal{B}_{1} \cap \mathcal{B}_{2}$ and $\left|\tilde{B}_{1} \cap \tilde{B}_{2}\right|<\left|B_{1} \cap B_{2}\right|$. This contradicts that $\left|B_{1} \cap B_{2}\right|$ is minimized.

We now consider the digraph $D=(V, E)$ defined by (1). For $x \in B_{1} \cap B_{2}$ and $y \in$ $S \backslash\left(B_{1} \cup B_{2}\right)$, we observe that $B_{1}-x+y \in \mathcal{B}_{1}$ implies $X_{0}-x+y \in 2 \mathcal{B}_{1}^{+}$and $B_{2}-x+y \in \mathcal{B}_{2}$ implies $X_{0}-x+y \in 2 \mathcal{B}_{2}^{+}$. This shows that $D$ is a subgraph of $D^{+}$, and hence $D$ is acyclic by Claim 20. Therefore, we can find a function $p: S \rightarrow \mathbb{R}$ such that $p(x):=0$ for $x \in B_{1} \backslash B_{2}, p(x):=|S|+1$ for $x \in B_{2} \backslash B_{1}, p(x)$ are distinct values in $\{1,2, \ldots,|S|\}$ for $x \in V$, and $p(x)<p(y)$ for $(x, y) \in E$. Then, Claim 16 shows that $\arg \min _{X \in \mathcal{B}_{1}} p(X)=\left\{B_{1}\right\}$ and $\arg \max _{X \in \mathcal{B}_{2}} p(X)=\left\{B_{2}\right\}$. Since $B_{1}, B_{2} \in \mathcal{B}_{1} \cap \mathcal{B}_{2}, p$ satisfies the requirements in Conjecture 11. Thus, Conjecture 11 holds.

This proof can be converted to a polynomial-time algorithm for computing $p$ as follows. We first pick up two arbitrary common bases $B_{1}, B_{2} \in \mathcal{B}_{1} \cap \mathcal{B}_{2}$ and construct a digraph $D^{+}$ as above. If $D^{+}$is acyclic, then we can find an appropriate function $p$. Otherwise, the proof of Claim 20 shows that we can find $\tilde{B}_{1}, \tilde{B}_{2} \in \mathcal{B}_{1} \cap \mathcal{B}_{2}$ with $\left|\tilde{B}_{1} \cap \tilde{B}_{2}\right|<\left|B_{1} \cap B_{2}\right|$. Then, we update $B_{i} \leftarrow \tilde{B}_{i}$ for $i \in\{1,2\}$, construct $D^{+}$, and repeat this procedure. Since $\left|B_{1} \cap B_{2}\right|$ decreases monotonically, this procedure is executed at most $|S|$ times.

Recall that Conjectures 2 and 11 are equivalent by replacing $M_{2}$ with $M_{2}^{*}$. Since $M_{2}$ is strongly base orderable if and only if $M_{2}^{*}$ is strongly base orderable, Conjecture 2 also holds for strongly base orderable matroids.

This together with Lemma 14 shows that Conjecture 1 also holds. We note that, if $M_{1}$ and $M_{2}$ are strongly base orderbale matroids, then the matroids $M_{1}^{\prime}$ and $M_{2}^{\prime}$ obtained by deletion and contraction in the proof of Lemma 14 are also strongly base orderable. 
Finally in this section, we show an application of Theorem 4 to bipartite matching, which is of independent interest. For a vertex $v$ in a graph, let $\delta(v)$ denote the set of all the edges incident to $v$.

- Corollary 21. For a bipartite graph $G=(U, V ; E)$ containing a perfect matching, there exists a weight function $w: E \rightarrow \mathbb{R}$ satisfying the following conditions.

1. For each $u \in U$, let $e_{u}$ be a lightest edge in $\delta(u)$ with respect to $w$. Then, $\left\{e_{u} \mid u \in U\right\}$ is a perfect matching in $G$.

2. For each $v \in V$, let $e_{v}$ be a heaviest edge in $\delta(v)$ with respect to $w$. Then, $\left\{e_{v} \mid v \in V\right\}$ is a perfect matching in $G$.

Proof. Let $\mathcal{B}_{1}=\{F \subseteq E|| F \cap \delta(u) \mid=1$ for any $u \in U\}$ and $\mathcal{B}_{2}=\{F \subseteq E|| F \cap \delta(v) \mid=$ 1 for any $v \in V\}$. By definition, $\left(E, \mathcal{B}_{1}\right)$ and $\left(E, \mathcal{B}_{2}\right)$ are partition matroids, and hence they are strongly base orderable matroids. Since Conjecture 11 holds for strongly base orderable matroids and $\mathcal{B}_{1} \cap \mathcal{B}_{2}$ is the set of perfect matchings in $G$, we obtain the corollary.

\section{Reduction from the weighted case to the unweighted case}

In this section, we show that the weighted problem can be reduced to the unweighted one, and prove Theorem 6 .

- Theorem 6. If Conjecture 2 is true, then Conjecture 5 is also true.

Proof. Since Conjectures 2 and 11 are equivalent, it suffices to show that Conjecture 5 is true by assuming that Conjecture 11 is true.

Suppose that we are given $M_{i}=\left(S, \mathcal{B}_{i}\right)$ and $w_{i}: S \rightarrow \mathbb{R}$ for $i \in\{1,2\}$ as in Conjecture 5 . We first consider the problem of finding a maximum weight common basis of $M_{1}$ and $M_{2}^{*}$ with respect to $w_{1}-w_{2}$, where $M_{2}^{*}=\left(S, \mathcal{B}_{2}^{*}\right)$ is the dual matroid of $M_{2}$. By Theorem 10, there exist two functions $q_{1}: S \rightarrow \mathbb{R}$ and $q_{2}: S \rightarrow \mathbb{R}$ with $q_{1}+q_{2}=w_{1}-w_{2}$ such that

$$
\underset{X \in \mathcal{B}_{1} \cap \mathcal{B}_{2}^{*}}{\arg \max }\left(w_{1}(X)-w_{2}(X)\right)=\left(\underset{X \in \mathcal{B}_{1}}{\arg \max } q_{1}(X)\right) \cap\left(\underset{X \in \mathcal{B}_{2}^{*}}{\arg \max } q_{2}(X)\right) .
$$

Define $\hat{\mathcal{B}}_{1}=\arg \max _{X \in \mathcal{B}_{1}} q_{1}(X)$ and $\hat{\mathcal{B}}_{2}=\arg \max _{X \in \mathcal{B}_{2}^{*}} q_{2}(X)$. Then, it is known that $\hat{M}_{i}=\left(S, \hat{\mathcal{B}}_{i}\right)$ is also a matroid for $i \in\{1,2\}$ (see [14]). By (2), we obtain

$$
\underset{X \in \mathcal{B}_{1} \cap \mathcal{B}_{2}^{*}}{\arg \max }\left(w_{1}(X)-w_{2}(X)\right)=\hat{\mathcal{B}_{1}} \cap \hat{\mathcal{B}_{2}} .
$$

This together with $\mathcal{B}_{1} \cap \mathcal{B}_{2}^{*} \neq \emptyset$ shows that $\hat{\mathcal{B}}_{1} \cap \hat{\mathcal{B}}_{2} \neq \emptyset$, and hence $\hat{M}_{1}$ and $\hat{M}_{2}$ satisfy the assumptions in Conjecture 11. Therefore, by assuming that Conjecture 11 is true, there exists a function $\hat{p}: S \rightarrow \mathbb{R}$ satisfying the following conditions.

(a) For any $B_{1} \in \arg \min _{X \in \hat{\mathcal{B}}_{1}} \hat{p}(X)$, it holds that $B_{1} \in \hat{\mathcal{B}}_{2}$.

(b) For any $B_{2} \in \arg \max _{X \in \hat{\mathcal{B}}_{2}} \hat{p}(X)$, it holds that $B_{2} \in \hat{\mathcal{B}}_{1}$.

Let $\delta:=\min \left\{\left|q_{i}(X)-q_{i}(Y)\right| \mid i \in\{1,2\}, X, Y \subseteq S, q_{i}(X) \neq q_{i}(Y)\right\}$ and let $\varepsilon$ be a positive number such that $\varepsilon \cdot|\hat{p}(X)|<\delta / 2$ for any $X \subseteq S$. We now show that $p:=w_{1}-q_{1}+\varepsilon \cdot \hat{p}$ satisfies the requirements of Conjecture 5 . Let $B_{1}$ be a set in $\arg \max _{X \in \mathcal{B}_{1}}\left(w_{1}(X)-p(X)\right)=$ $\arg \max _{X \in \mathcal{B}_{1}}\left(q_{1}(X)-\varepsilon \cdot \hat{p}(X)\right)$. Since $-\delta / 2<\varepsilon \cdot \hat{p}(X)<\delta / 2$ for any $X \subseteq S$, we have that $B_{1} \in \arg \max _{X \in \mathcal{B}_{1}} q_{1}(X)=\hat{\mathcal{B}}_{1}$ and $B_{1} \in \arg \min _{X \in \hat{\mathcal{B}_{1}}} \hat{p}(X)$. Then (a) shows that $B_{1} \in \hat{\mathcal{B}_{2}}$. 
Therefore,

$$
B_{1} \in \hat{\mathcal{B}_{1}} \cap \hat{\mathcal{B}_{2}}=\underset{X \in \mathcal{B}_{1} \cap \mathcal{B}_{2}^{*}}{\arg \max }\left(w_{1}(X)-w_{2}(X)\right)=\underset{X \in \mathcal{B}_{1} \cap \mathcal{B}_{2}^{*}}{\arg \max }\left(w_{1}(X)+w_{2}(S \backslash X)\right)
$$

holds by (3), which means that $p$ satisfies the first requirement in Conjecture 5.

Similarly, let $B_{2}$ be a set in

$$
\underset{X \in \mathcal{B}_{2}}{\arg \max }\left(w_{2}(X)-p(X)\right)=\underset{X \in \mathcal{B}_{2}}{\arg \max }\left(-q_{2}(X)-\varepsilon \cdot \hat{p}(X)\right)=\underset{X \in \mathcal{B}_{2}}{\arg \max }\left(q_{2}(S \backslash X)+\varepsilon \cdot \hat{p}(S \backslash X)\right) .
$$

This shows that $S \backslash B_{2} \in \arg \max _{X \in \mathcal{B}_{2}^{*}} q_{2}(X)=\hat{\mathcal{B}_{2}}$ and $S \backslash B_{2} \in \arg \max _{X \in \hat{\mathcal{B}_{2}}} \hat{p}(X)$. Then (b) shows that $S \backslash B_{2} \in \hat{\mathcal{B}_{1}}$. Therefore,

$$
S \backslash B_{2} \in \hat{\mathcal{B}_{1}} \cap \hat{\mathcal{B}_{2}}=\underset{X \in \mathcal{B}_{1} \cap \mathcal{B}_{2}^{*}}{\arg \max }\left(w_{1}(X)-w_{2}(X)\right)=\underset{X \in \mathcal{B}_{1} \cap \mathcal{B}_{2}^{*}}{\arg \max }\left(w_{1}(X)+w_{2}(S \backslash X)\right)
$$

holds by (3), which means that $p$ satisfies the second requirement in Conjecture 5. Therefore, Conjecture 5 is true if Conjecture 11 is true.

- Remark 22. Algorithmically, if we can compute $\hat{p}$, then we can compute $p$ efficiently as follows. Since $w_{1}$ and $w_{2}$ are rational-valued when they are given as a part of input, by multiplying by the common denominator, we may assume that $w_{1}$ and $w_{2}$ are integral. Then, we can take $q_{1}$ and $q_{2}$ so that they are integral [18]. Therefore, we have that $\delta \geq 1$, and hence $\varepsilon:=1 /\left(1+2 \sum_{s \in S}|\hat{p}(s)|\right)$ satisfies the conditions in the proof. This shows that we can compute $p:=w_{1}-q_{1}+\varepsilon \cdot \hat{p}$.

By Theorem 6, we obtain Corollaries 7 and 8 as follows. In the proof of Theorem 6 , we consider Conjecture 11 for matroids $\hat{M}_{i}=\left(S, \hat{\mathcal{B}}_{i}\right)$, where $\hat{\mathcal{B}}_{1}=\arg \max _{X \in \mathcal{B}_{1}} q_{1}(X)$ and $\hat{\mathcal{B}_{2}}=\arg \max _{X \in \mathcal{B}_{2}^{*}} q_{2}(X)$. Observe that if $M_{1}$ is a partition matroid with partition classes of size at most 2 and with all-ones upper bound on the partition classes, then so is $\hat{M}_{1}$. Furthermore, Lemma 13 shows that if $M_{i}$ is strongly base orderable, then so is $\hat{M}_{i}$. Since Theorems 3 and 4 imply that Conjecture 11 also holds for these cases, we obtain Corollaries 7 and 8 .

\section{Conclusion}

We considered the existence of prices that are capable of achieving optimal social welfare without a central tie-breaking coordinator. Although such pricing looks similar to well-known Walrasian pricing, it is less understood even for two-buyer markets with gross substitute valuations. This paper focuses on two-buyer markets with matroid rank valuations, and we gave polynomial-time algorithms that always find such prices when one of the matroids is a simple partition matroid or both matroids are strongly base orderable. This result partially answers a question of Düetting and Végh. We further formalized a weighted variant of the conjecture of Düetting and Végh, and showed that the weighted variant can be reduced to the unweighted one based on the weight-splitting theorem of Frank. We also showed that a similar reduction technique works for $\mathrm{M}^{\natural}$-concave functions, or equivalently, for gross substitutes functions (see [1] for the details). 


\section{References}

1 Kristóf Bérczi, Naonori Kakimura, and Yusuke Kobayashi. Market pricing for matroid rank valuations. arXiv preprint, 2020. arXiv:2007.08759.

2 Ben Berger, Alon Eden, and Michal Feldman. On the power and limits of dynamic pricing in combinatorial markets. arXiv preprint, 2020. arXiv:2002.06863.

3 Liad Blumrosen and Thomas Holenstein. Posted prices vs. negotiations: an asymptotic analysis. EC, 10:1386790-1386801, 2008.

4 Shuchi Chawla, Jason D. Hartline, David L. Malec, and Balasubramanian Sivan. Multiparameter mechanism design and sequential posted pricing. In Proceedings of the Forty-Second ACM Symposium on Theory of Computing, pages 311-320, 2010.

5 Shuchi Chawla, David L. Malec, and Balasubramanian Sivan. The power of randomness in Bayesian optimal mechanism design. In Proceedings of the 11th ACM Conference on Electronic Commerce, pages 149-158, 2010.

6 Shuchi Chawla, J Benjamin Miller, and Yifeng Teng. Pricing for online resource allocation: intervals and paths. In Proceedings of the Thirtieth Annual ACM-SIAM Symposium on Discrete Algorithms, pages 1962-1981. SIAM, 2019.

7 Edward H Clarke. Multipart pricing of public goods. Public choice, pages 17-33, 1971.

8 Vincent Cohen-Addad, Alon Eden, Michal Feldman, and Amos Fiat. The invisible hand of dynamic market pricing. In Proceedings of the 2016 ACM Conference on Economics and Computation, pages 383-400, 2016.

9 Joan Davies and Colin McDiarmid. Disjoint common transversals and exchange structures. Journal of the London Mathematical Society, 2(1):55-62, 1976.

10 Paul Dütting, Michal Feldman, Thomas Kesselheim, and Brendan Lucier. Posted prices, smoothness, and combinatorial prophet inequalities. arXiv preprint, 2016. arXiv:1612.03161.

11 Paul Dütting, Michal Feldman, Thomas Kesselheim, and Brendan Lucier. Prophet inequalities made easy: Stochastic optimization by pricing non-stochastic inputs. In 2017 IEEE 58th Annual Symposium on Foundations of Computer Science (FOCS), pages 540-551. IEEE, 2017.

12 Paul Dütting and Lśzló A. Végh. Private Communication, 2017.

13 Alon Eden, Uriel Feige, and Michal Feldman. Max-min greedy matching. Approximation, Randomization, and Combinatorial Optimization. Algorithms and Techniques (APPROX/RANDOM 2019), 145:7, 2019.

14 Jack Edmonds. Matroids and the greedy algorithm. Mathematical Programming, 1(1):127-136, 1971.

15 Tomer Ezra, Michal Feldman, Tim Roughgarden, and Warut Suksompong. Pricing multi-unit markets. In International Conference on Web and Internet Economics, pages 140-153. Springer, 2018.

16 Michal Feldman, Nick Gravin, and Brendan Lucier. Combinatorial auctions via posted prices. In Proceedings of the Twenty-Sixth Annual ACM-SIAM Symposium on Discrete Algorithms, pages 123-135. SIAM, 2014.

17 Michal Feldman, Nick Gravin, and Brendan Lucier. Combinatorial Walrasian equilibrium. SIAM Journal on Computing, 45(1):29-48, 2016.

18 András Frank. A weighted matroid intersection algorithm. Journal of Algorithms, 2(4):328-336, 1981.

19 Theodore Groves. Incentives in teams. Econometrica: Journal of the Econometric Society, pages $617-631,1973$.

20 Faruk Gul and Ennio Stacchetti. Walrasian equilibrium with gross substitutes. Journal of Economic theory, 87(1):95-124, 1999.

21 Justin Hsu, Jamie Morgenstern, Ryan Rogers, Aaron Roth, and Rakesh Vohra. Do prices coordinate markets? In Proceedings of the Forty-Eighth Annual ACM Symposium on Theory of Computing, pages 440-453, 2016.

22 Rosa Huang and Gian-Carlo Rota. On the relations of various conjectures on latin squares and straightening coefficients. Discrete Mathematics, 128(1-3):225-236, 1994. 
23 Alexander S. Kelso Jr and Vincent P. Crawford. Job matching, coalition formation, and gross substitutes. Econometrica: Journal of the Econometric Society, pages 1483-1504, 1982.

24 Stein Krogdahl. A combinatorial base for some optimal matroid intersection algorithms. Technical Report STAN-CS-74-468, Computer Science Department, Stanford University, Stanford, CA, U.S., 1974.

25 Stein Krogdahl. A combinatorial proof for a weighted matroid intersection algorithm. Technical Report Computer Science Report 17, Institute of Mathematical and Physical Sciences, University of Tromso, Tromso, Norway, 1976.

26 Stein Krogdahl. The dependence graph for bases in matroids. Discrete Mathematics, 19(1):47$59,1977$.

27 Noam Nisan and Ilya Segal. The communication requirements of efficient allocations and supporting prices. Journal of Economic Theory, 129(1):192-224, 2006.

28 Hirokazu Nishimura and Susumu Kuroda. A Lost Mathematician, Takeo Nakasawa: The Forgotten Father of Matroid Theory. Springer Science \& Business Media, 2009.

29 James Oxley. Matroid Theory. Oxford University Press, 2011.

30 Alexander Schrijver. Combinatorial Optimization: Polyhedra and Efficiency, volume 24. Springer Science \& Business Media, 2003.

31 William Vickrey. Counterspeculation, auctions, and competitive sealed tenders. The Journal of Finance, 16(1):8-37, 1961.

32 Léon Walras. Éléments d'économie politique pure, ou, Théorie de la richesse sociale. F. Rouge, 1896.

33 Hassler Whitney. On the abstract properties of linear dependence. In Hassler Whitney Collected Papers, pages 147-171. Springer, 1992. 